PROCEEDINGS OF THE

AMERICAN MATHEMATICAL SOCIETY

Volume 128, Number 4, Pages 1063-1070

S 0002-9939(99)05092-3

Article electronically published on July 27, 1999

\title{
ON A POLYNOMIAL INEQUALITY OF E. J. REMEZ
}

\author{
D. DRYANOV AND Q. I. RAHMAN \\ (Communicated by Frederick W. Gehring)
}

\begin{abstract}
We prove a result which extends a well-known polynomial inequality of E. J. Remez and another one due to W. A. Markov.
\end{abstract}

\section{INTRODUCTION}

Let $\mathcal{P}_{n}$ denote the class of all polynomials of degree at most $n$ with real or complex coefficients. Polynomials in $\mathcal{P}_{n}$ whose coefficients are all real will form the sub-class $\mathcal{P}_{n, \mathbb{R}}$. As usual we shall denote by $T_{n}$ the $n$th Chebyshev polynomial of the first kind, which is given by $\cos n \arccos x$ for $-1 \leq x \leq 1$. In particular, $\left|T_{n}(x)\right| \leq 1$ for $-1 \leq x \leq 1$ and $T_{n}(\cos ((n-j) \pi / n))=(-1)^{n-j}$ for $j=0,1, \ldots, n$. All its zeros are real and lie in the open interval $(-1,1)$. It was observed by P.L. Chebyshev (see [7] or [9]) that if $f \in \mathcal{P}_{n}$ and $|f(x)| \leq 1$ for $-1 \leq x \leq 1$, then

$$
|f(x)| \leq\left|T_{n}(x)\right| \text { for all } x \in \mathbb{R} \backslash[-1,1] .
$$

Subsequently, it was shown by W.A. Markov [5] that under the same condition on $f$, we have

$$
\left|f^{(k)}(x)\right| \leq\left|T_{n}^{(k)}(x)\right| \text { for all } x \in \mathbb{R} \backslash[-1,1] \text { and } 1 \leq k \leq n .
$$

Now we must introduce a couple of additional notations. We shall write $\mu(\mathfrak{S})$ for the measure of a Lebesgue measurable subset $\mathfrak{S}$ of $\mathbb{R}$. For any polynomial $f$ and any subinterval $\mathbb{I}$ of $\mathbb{R}$ we denote the set $\{x \in \mathbb{I}:|f(x)| \leq 1\}$ by $\mathfrak{E}(f ; \mathbb{I})$.

The following generalization of Chebyshev's inequality (1) is due to E.J. Remez (see [1], 2], [3] Lemma 7.3], [8]). The proof in [1] is the simplest.

Theorem A. For all $g \in \mathcal{P}_{n}$, the following inequality holds:

$$
\max _{-1 \leq x \leq 1}|g(x)| \leq T_{n}\left(\frac{4}{\mu(\mathfrak{E}(g ;[-1,1]))}-1\right) .
$$

An equivalent formulation of this result stated below as Theorem A' shows clearly why it contains (1).

Received by the editors May 12, 1997 and, in revised form, May 25, 1998.

1991 Mathematics Subject Classification. Primary 30A10, 30C10, 41A17.

Key words and phrases. Polynomials, Markov's inequality, Remez inequality.

The first author was partially supported by the Bulgarian Ministry of Education, Sciences, and Technology through Contract 513/95. 
For each $R \geq 1$, let

$$
\begin{gathered}
\pi_{n}(R):=\left\{f \in \mathcal{P}_{n}: \mu(\mathfrak{E}(f ;[-1, R])) \geq 2\right\}, \\
\pi_{n, \mathbb{R}}(R):=\left\{f \in \mathcal{P}_{n, \mathbb{R}}: \mu(\mathfrak{E}(f ;[-1, R])) \geq 2\right\} .
\end{gathered}
$$

If $f \in \pi_{n}(R)$ for some $R \geq 1$ and $g(x):=f((R+1) x / 2+(R-1) / 2)$, then

$$
\mu(\mathfrak{E}(g ;[-1,1]))=\frac{2}{R+1} \mu(\mathfrak{E}(f ;[-1, R])) \geq \frac{4}{R+1} .
$$

Hence Theorem A implies that if $f \in \pi_{n}(R)$, then

$$
\max _{-1 \leq x \leq R}|f(x)|=\max _{-1 \leq x \leq 1}|g(x)| \leq T_{n}(R) .
$$

Conversely, let (4) hold for all $f \in \pi_{n}(R)$. If $g$ is any polynomial of degree at most $n$ and $f(x):=g((2 x-R+1) /(R+1))$, then

$$
\mu(\mathfrak{E}(f ;[-1, R]))=\frac{R+1}{2} \mu(\mathfrak{E}(g ;[-1,1])) \geq 2
$$

if $R \geq 4 / \mu(\mathfrak{E}(g ;[-1,1]))-1$. Hence, $f \in \pi_{n}(R)$ for all such values of $R$ and

$$
\max _{-1 \leq x \leq 1}|g(x)|=\max _{-1 \leq x \leq R}|f(x)| \leq T_{n}(R),
$$

that is, (3) holds. Thus, Theorem A may be reformulated as follows.

Theorem $\mathbf{A}^{\prime}$. If $f \in \pi_{n}(R)$ for some $R \geq 1$, then

$$
\max _{-1 \leq x \leq R}|f(x)| \leq T_{n}(R) .
$$

It was noted by B.D. Bojanov that if $m(t):=\sup \left\{|f(t)|: f \in \pi_{n}(R)\right\}$, then

$$
m(t) \leq m(R)
$$

for all $t \in(-1, R)$. His argument goes roughly as follows.

Take an arbitrary $t \in(-1, R)$ and any $f \in \pi_{n}(R)$. If

$$
q_{1}(t):=\frac{\mu(\mathfrak{E}(f ;[-1, t]))}{1+t} \quad \text { and } \quad q_{2}(t):=\frac{\mu(\mathfrak{E}(f ;[t, R]))}{R-t}
$$

then

$$
\max \left\{q_{1}(t), q_{2}(t)\right\} \geq \frac{2}{1+R},
$$

since otherwise, we would have

$$
\mu(\mathfrak{E}(f ;[-1, R]))=\mu(\mathfrak{E}(f ;[-1, t]))+\mu(\mathfrak{E}(f ;[t, R]))<2 .
$$

Now consider the linear transformations

$$
\alpha_{1}(x):=\frac{(1+t) x-R+t}{1+R} \text { and } \quad \alpha_{2}(x):=\frac{(t-R) x+R^{2}+t}{1+R} .
$$

It is to be noted that as $x$ increases from -1 to $R$, the number $\alpha_{1}(x)$ increases from -1 to $t$ whereas $\alpha_{2}(x)$ decreases from $R$ to $t$. Under the first transformation every subinterval of $[-1, R]$ shrinks by the factor $(1+t) /(1+R)$; under the second, they all shrink by the factor $(R-t) /(1+R)$. This means that if $\mathbb{I}$ is an interval contained either in $[-1, t]$ or in $[t, R]$, then $\mu\left(\left\{x \in[-1, R]: \alpha_{1}(x) \in \mathbb{I}\right\}\right)$ is equal to $((1+R) /(1+t)) \mu(\mathbb{I})$ in the first case and $\mu\left(\left\{x \in[-1, R]: \alpha_{2}(x) \in \mathbb{I}\right\}\right)$ is equal 
to $((1+R) /(R-t)) \mu(\mathbb{I})$ in the second. Hence, choosing $\kappa \in\{1,2\}$ such that $q_{\kappa}(t) \geq 2 /(1+R)$ we obtain

$$
\mu\left(\mathfrak{E}\left(f\left(\alpha_{\kappa}(\cdot)\right) ;[-1, R]\right)\right) \geq 2 .
$$

In other words, $f\left(\alpha_{\kappa}(\cdot)\right) \in \pi_{n}(R)$. Since $t=\alpha_{\kappa}(R)$, we conclude that

$$
|f(t)|=\left|f\left(\alpha_{\kappa}(R)\right)\right| \leq m(R) .
$$

In view of this fact, Theorem $\mathrm{A}^{\prime}$ may be stated as follows.

Theorem $\mathbf{A}^{\prime \prime}$. Let $R \geq 1$. If $f \in \pi_{n}(R)$, then for all $x \geq R$,

$$
|f(x)| \leq T_{n}(x) .
$$

We prove

Theorem 1. Let $R \geq 1$. If $f \in \pi_{n, \mathbb{R}}(R)$, then for all $z \in \mathcal{H}_{R}:=\{z \in \mathbb{C}: \operatorname{Re}(z) \geq$ $R\}$ we have

$$
\left|f^{(k)}(z)\right| \leq\left|T_{n}^{(k)}(z)\right| \quad(k=0,1, \ldots, n) .
$$

If $k$ belongs to $\{1, \ldots, n\}$, then equality holds in (7) for any $z \in \mathcal{H}_{R}$ if and only if $f(z)= \pm T_{n}(z)$. The same can be said when $k=0$ if $z \in \mathcal{H}_{R} \backslash\{1\}$.

Theorem 1 is not only an extension of Theorem $\mathrm{A}^{\prime \prime}$ but also of (2).

Note that if $f$ is of degree $n$, then $\mathfrak{E}(f ;(-\infty, \infty))$ consists of at most $n$ disjoint closed though possibly degenerate intervals. To see this consider the polynomial $F(z):=f(z) \overline{f(\bar{z})}$. It is non-negative on the real axis and $\mathfrak{E}(F ;(-\infty, \infty))=$ $\mathfrak{E}(f ;(-\infty, \infty))$. Suppose that $\mathfrak{E}(f ;(-\infty, \infty))$ consists of $N$ disjoint closed intervals $\left[a_{1}, b_{1}\right], \ldots,\left[a_{N}, b_{N}\right]$, where $N \geq n+1$. It is geometrically evident that the derivative $F^{\prime}$ must vanish at least once in each of the $N-1$ open intervals $\left(b_{1}, a_{2}\right), \ldots,\left(b_{N-1}, a_{N}\right)$ and also in each of the intervals $\left[a_{1}, b_{1}\right], \ldots,\left[a_{N}, b_{N}\right]$, even in the degenerate ones. Thus $F^{\prime}$ has at least $2 N-1(\geq 2 n+1)$ zeros, which is a contradiction since $F^{\prime}$ is of degree $2 n-1$. It follows that $\mathfrak{E}(f ;[-1, \xi])$ consists of at most $n$ disjoint, closed, possibly degenerate intervals for all $\xi \geq-1$.

Now let $x_{j}:=\cos ((n-j) \pi / n)$ for $j=0,1, \ldots, n$ and let $f$ be an arbitrary polynomial in $\pi_{n}(R)$, where $R \geq 1$. For $j=0,1, \ldots, n$ let $\xi_{j}$ be the infimum of all $\xi$ such that $\mu(\mathfrak{E}(f ;[-1, \xi]))=1+x_{j}$. The numbers $\xi_{0}, \xi_{1}, \ldots, \xi_{n}$ are well defined and form an increasing sequence such that $\xi_{j+1}-\xi_{j} \geq x_{j+1}-x_{j}$ for $j=0, \ldots, n-1$; in particular, $\xi_{j} \geq x_{j}$ for $j=0,1, \ldots, n$. So, Theorem 1 is contained in the following result. This is what we shall really prove.

Theorem 1*. Let

$$
x_{j}:=\cos \frac{n-j}{n} \pi \quad \text { for } j=0,1, \ldots, n .
$$

Further, let $\xi_{0}, \xi_{1}, \ldots, \xi_{n}$ be another sequence of $n+1$ numbers in $[-1, \infty)$ such that

$$
\xi_{j+1}-\xi_{j} \geq x_{j+1}-x_{j} \quad(j=0,1, \ldots, n-1),
$$

and $\mathcal{H}_{R}$ as in Theorem 1. If $f$ is a real polynomial of degree at most $n$ such that

$$
\left|f\left(\xi_{j}\right)\right| \leq 1 \quad(j=0,1, \ldots, n),
$$

then for all $z \in \mathcal{H}_{R}$ with $R \geq \xi_{n}$ we have

$$
\left|f^{(k)}(z)\right| \leq\left|T_{n}^{(k)}(z)\right| \quad(k=0,1, \ldots, n),
$$


where $f^{(0)}(z) \equiv f(z)$. If $k$ belongs to $\{1, \ldots, n\}$, then equality holds in (10) for any $z \in \mathcal{H}_{R}$ if and only if $\xi_{j}=x_{j}$ for all $j$ and $f(z)= \pm T_{n}(z)$. The same can be said when $k=0$ if $z \in \mathcal{H}_{R} \backslash\{1\}$.

Remark 1. Let $f$ be any polynomial (real or not) of degree at most $n$ satisfying (9). For any $x_{0} \geq \xi_{n}$ and any $k \in\{0,1, \ldots, n\}$, let $f^{(k)}\left(x_{0}\right)=\left|f^{(k)}\left(x_{0}\right)\right| e^{i \gamma}$. Then $g(x):=\operatorname{Re}\left(e^{-i \gamma} f(x)\right)$ is a real polynomial of degree at most $n$ satisfying (9) and so

$$
\left|f^{(k)}\left(x_{0}\right)\right|=\operatorname{Re}\left(e^{-i \gamma} f^{(k)}\left(x_{0}\right)\right)=\left|g^{(k)}\left(x_{0}\right)\right| \leq T_{n}^{(k)}\left(x_{0}\right)
$$

by (10). In other words, (10) holds for any polynomial $f$ of degree at most $n$ satisfying (9) if $z \in \mathcal{H}_{R} \cap \mathbb{R}$.

\section{Preparatory lemmas}

The following auxiliary result is a simple principle of mechanics expressed in terms of complex numbers rather than vectors.

Lemma 1. Let $\varphi_{1}, \ldots, \varphi_{n}$ and $\psi_{1}, \ldots, \psi_{n}$ be non-negative numbers with $\varphi_{k} \leq \psi_{k}$ for $k=1, \ldots, n$ and $\sum_{k=1}^{n} \psi_{k}<\pi / 2$. Besides, let $\rho_{0}, \ldots, \rho_{n}$ and $R_{0}, \ldots, R_{n}$ be two other sets of positive numbers such that $\rho_{k} \leq R_{k}$ for $k=0,1, \ldots, n$. Then

$$
\left|\rho_{0}+\sum_{k=1}^{n} \rho_{k} \exp \left(-i \sum_{j=1}^{k} \psi_{j}\right)\right| \leq\left|R_{0}+\sum_{k=1}^{n} R_{k} \exp \left(-i \sum_{j=1}^{k} \varphi_{j}\right)\right|,
$$

where equality holds only if $\rho_{k}=R_{k}$ for $0 \leq k \leq n$ and $\psi_{k}=\varphi_{k}$ for $1 \leq k \leq n$.

Proof. Clearly, $\left|\rho_{0}+\sum_{k=1}^{n} \rho_{k} \exp \left(-i \sum_{j=1}^{k} \psi_{j}\right)\right|^{2}$ is equal to

$$
\begin{aligned}
\sum_{k=0}^{n} \rho_{k}^{2}+2 \rho_{0} \sum_{l=1}^{n} \rho_{l} \cos \left(\sum_{j=1}^{l} \psi_{j}\right)+ & \sum_{k=1}^{n-1} 2 \rho_{k} \cos \left(\sum_{j=1}^{k} \psi_{j}\right)\left(\sum_{l=k+1}^{n} \rho_{l} \cos \left(\sum_{j=1}^{l} \psi_{j}\right)\right) \\
& +\sum_{k=1}^{n-1} 2 \rho_{k} \sin \left(\sum_{j=1}^{k} \psi_{j}\right)\left(\sum_{l=k+1}^{n} \rho_{l} \sin \left(\sum_{j=1}^{l} \psi_{j}\right)\right),
\end{aligned}
$$

which is in turn equal to

$$
\begin{aligned}
\sum_{k=0}^{n} \rho_{k}^{2}+2 \rho_{0} \sum_{l=1}^{n} \rho_{l} & \cos \left(\sum_{j=1}^{l} \psi_{j}\right)+\sum_{k=1}^{n-1} 2 \rho_{k} \sum_{l=k+1}^{n} \rho_{l} \\
\times & \left\{\cos \left(\sum_{j=1}^{l} \psi_{j}\right) \cos \left(\sum_{j=1}^{k} \psi_{j}\right)+\sin \left(\sum_{j=1}^{l} \psi_{j}\right) \sin \left(\sum_{j=1}^{k} \psi_{j}\right)\right\} .
\end{aligned}
$$

Thus, $\left|\rho_{0}+\sum_{k=1}^{n} \rho_{k} \exp \left(-i \sum_{j=1}^{k} \psi_{j}\right)\right|^{2}$ can be written in the form

$$
\sum_{k=0}^{n} \rho_{k}^{2}+\sum_{k=0}^{n-1} 2 \rho_{k} \sum_{l=k+1}^{n} \rho_{l} \cos \left(\sum_{j=k+1}^{l} \psi_{j}\right)
$$

which, obviously, increases as any of the numbers $\rho_{0}, \rho_{1}, \ldots, \rho_{n}$ increases or as any of the numbers $\psi_{1}, \ldots, \psi_{n}$ decreases. Hence (11) holds, wherein equality holds only if $\rho_{k}=R_{k}$ for $0 \leq k \leq n$ and $\psi_{k}=\varphi_{k}$ for $1 \leq k \leq n$. 
Lemma 2. Let $x_{j}, \xi_{j}$ be as in Theorem $1^{*}$ and $z=x+$ iy where $x \geq \xi_{n}, y \geq 0$. Denote by $A_{j}, B_{j}$ and $P$ the points of the complex plane which correspond to $x_{j}, \xi_{j}$ and $z$, respectively. If $\varphi_{j}, \psi_{j}$ stand for the angles $\widehat{A_{j-1} P} A_{j}, \widehat{B_{j-1} P B_{j}}$, respectively, then

$$
\psi_{j} \geq \varphi_{j} \text { for } j=1, \ldots, n,
$$

where, in the case $y>0$, equality holds for some $j$ if and only if $\xi_{j}=x_{j}$ for all $j$.

Proof. There is nothing to prove when $y=0$ since in that case $\psi_{j}$ and $\varphi_{j}$ are all zero. So we assume $y>0$. For $j=1, \ldots, n$ let $\delta_{j}, \Delta_{j}$ denote the areas of the triangles $A_{j-1} P A_{j}, B_{j-1} P B_{j}$, respectively; then

$$
\delta_{j}=\frac{1}{2}\left(x_{j}-x_{j-1}\right) y, \Delta_{j}=\frac{1}{2}\left(\xi_{j}-\xi_{j-1}\right) y .
$$

By assumption, $\xi_{j}-\xi_{j-1} \geq x_{j}-x_{j-1}$ and so

$$
\Delta_{j} \geq \delta_{j} \text { for } 1 \leq j \leq n .
$$

Using another well-known formula for the area of a triangle we write

$$
\delta_{j}=\frac{1}{2}\left|z-x_{j}\right|\left|z-x_{j-1}\right| \sin \varphi_{j}, \Delta_{j}=\frac{1}{2}\left|z-\xi_{j}\right|\left|z-\xi_{j-1}\right| \sin \psi_{j},
$$

from which, for $j=1, \ldots, n$, we obtain

$$
\sin \varphi_{j}=\frac{2 \delta_{j}}{\left|z-x_{j}\right|\left|z-x_{j-1}\right|}, \sin \psi_{j}=\frac{2 \Delta_{j}}{\left|z-\xi_{j}\right|\left|z-\xi_{j-1}\right|} .
$$

It is geometrically evident that $\left|z-\xi_{j}\right| \leq\left|z-x_{j}\right|$ for $j=0,1, \ldots, n$. Hence, (14) combined with (13) implies that

$$
\sin \varphi_{j} \leq \sin \psi_{j} \quad(1 \leq j \leq n) .
$$

This is equivalent to the desired result since $0<\varphi_{j}, \psi_{j}<\pi / 2$.

Lemma 3. Let $x_{j}, z$ and $\varphi_{j}$ be as in Lemma 2. If

$$
G(z):=\prod_{j=0}^{n}\left(z-x_{j}\right), G_{k}(z):=\frac{G(z)}{z-x_{k}} \text { for } k=0,1, \ldots, n,
$$

then with $\varphi_{0}=0$, we have

$$
\left|T_{n}(z)\right|=\left|\sum_{k=0}^{n} \frac{1}{\left|G^{\prime}\left(x_{k}\right)\right|}\right| G_{k}(z)\left|\exp \left(-i \sum_{j=0}^{k} \varphi_{j}\right)\right| .
$$

Proof. Note that

$$
G^{\prime}\left(x_{k}\right)=\prod_{j=0, j \neq k}^{n}\left(x_{k}-x_{j}\right)=(-1)^{n-k} \prod_{j=0, j \neq k}^{n}\left|x_{k}-x_{j}\right|=(-1)^{n-k}\left|G^{\prime}\left(x_{k}\right)\right|
$$

and

$$
T_{n}\left(x_{k}\right)=(-1)^{n-k} .
$$

Hence, by Lagrange interpolation in the points $x_{0}, x_{1}, \ldots, x_{n}$ we obtain

$$
\left|T_{n}(z)\right|=\left|\sum_{k=0}^{n} T_{n}\left(x_{k}\right) \frac{1}{G^{\prime}\left(x_{k}\right)} G_{k}(z)\right|=\left|\sum_{k=0}^{n} \frac{1}{\left|G^{\prime}\left(x_{k}\right)\right|} G_{k}(z)\right| .
$$


If $\alpha:=\operatorname{Arg} G_{0}(z)$ and $k \in\{0,1, \ldots, n\}$, then

$$
\begin{aligned}
G_{k}(z) & =G_{0}(z) \frac{z-x_{0}}{z-x_{k}} \\
& =e^{i \alpha}\left|G_{0}(z)\right| \frac{z-x_{0}}{z-x_{k}} \mid \exp \left(-i \sum_{j=0}^{k} \varphi_{j}\right) \\
& =e^{i \alpha}\left|G_{k}(z)\right| \exp \left(-i \sum_{j=0}^{k} \varphi_{j}\right)
\end{aligned}
$$

Substituting this expression for $G_{k}(z)$ in (16) we obtain (15).

Remark 2. Let $\xi_{j}, z$ and $\psi_{j}$ be as in Lemma 2. Further, let

$$
H(z):=\prod_{j=0}^{n}\left(z-\xi_{j}\right), H_{k}(z):=\frac{H(z)}{z-\xi_{k}} \text { for } k=0,1, \ldots, n .
$$

Arguing as for (17) we can show that if $\beta:=\operatorname{Arg} H_{0}(z)$, then with $\psi_{0}=0$, we have

$$
H_{k}(z)=e^{i \beta}\left|H_{k}(z)\right| \exp \left(-i \sum_{j=0}^{k} \psi_{j}\right) \text { for } k=0,1, \ldots, n .
$$

Lemma 4. For $k=0,1, \ldots, n$ let $w_{k}=u_{k}+i v_{k}$, where $u_{k}>0, v_{k} \leq 0$. If $-1 \leq t_{k} \leq 1$ for $k=0,1, \ldots, n$, then

$$
\left|\sum_{k=0}^{n} t_{k} w_{k}\right| \leq\left|\sum_{k=0}^{n} w_{k}\right|
$$

where equality holds if and only if the numbers $t_{k}$ are all of the same sign and of modulus 1.

Proof. Since the numbers $u_{k}$ are all of the same sign and so are the numbers $v_{k}$, we clearly have

$$
\begin{aligned}
\left|\sum_{k=0}^{n} t_{k} w_{k}\right|^{2} & =\left(\sum_{k=0}^{n} t_{k} u_{k}\right)^{2}+\left(\sum_{k=0}^{n} t_{k} v_{k}\right)^{2} \\
& \leq\left(\sum_{k=0}^{n} u_{k}\right)^{2}+\left(\sum_{k=0}^{n} v_{k}\right)^{2} \\
& =\left|\sum_{k=0}^{n} u_{k}+i \sum_{k=0}^{n} v_{k}\right|^{2} \\
& =\left|\sum_{k=0}^{n} w_{k}\right|^{2} .
\end{aligned}
$$




\section{Proof of Theorem $1^{*}$}

First let $k=0$. For reasons of symmetry it is enough to prove that

$$
|f(z)| \leq\left|T_{n}(z)\right| \text { for } z=x+i y, x \geq \xi_{n}, y \geq 0 .
$$

By Lagrange interpolation in the points $\xi_{0}, \xi_{1}, \ldots, \xi_{n}$ we have

$$
f(z)=\sum_{k=0}^{n} f\left(\xi_{k}\right) \frac{1}{H^{\prime}\left(\xi_{k}\right)} H_{k}(z) .
$$

Noting that

$$
H^{\prime}\left(\xi_{k}\right)=\prod_{j=0, j \neq k}^{n}\left(\xi_{k}-\xi_{j}\right)=(-1)^{n-k} \prod_{j=0, j \neq k}^{n}\left|\xi_{k}-\xi_{j}\right|=(-1)^{n-k}\left|H^{\prime}\left(\xi_{k}\right)\right|
$$

and taking (18) into account we obtain

$$
|f(z)|=\left|e^{i \beta} \sum_{k=0}^{n}(-1)^{n-k} f\left(\xi_{k}\right) \frac{\left|H_{k}(z)\right|}{\left|H^{\prime}\left(\xi_{k}\right)\right|} \exp \left(-i \sum_{j=0}^{k} \psi_{j}\right)\right| .
$$

So by Lemma 4 ,

$$
|f(z)| \leq\left|\sum_{k=0}^{n} \frac{\left|H_{k}(z)\right|}{\left|H^{\prime}\left(\xi_{k}\right)\right|} \exp \left(-i \sum_{j=0}^{k} \psi_{j}\right)\right| .
$$

From (8) it follows that $\left|\xi_{k}-\xi_{j}\right| \geq\left|x_{k}-x_{j}\right|$ and so

$$
\left|H^{\prime}\left(\xi_{k}\right)\right|=\prod_{j=0, j \neq k}^{n}\left|\xi_{k}-\xi_{j}\right| \geq \prod_{j=0, j \neq k}^{n}\left|x_{k}-x_{j}\right|=\left|G^{\prime}\left(x_{k}\right)\right| .
$$

Besides, it is geometrically evident that $\left|H_{k}(z)\right| \leq\left|G_{k}(z)\right|$. Since $\psi_{j} \geq \varphi_{j}$ by Lemma 2, we may apply Lemma 1 to conclude that for $z=x+i y$ with $x \geq \xi_{n}, y>0$ we have

$$
|f(z)| \leq\left|\sum_{k=0}^{n} \frac{\left|G_{k}(z)\right|}{\left|G^{\prime}\left(x_{k}\right)\right|} \exp \left(-i \sum_{j=0}^{k} \varphi_{j}\right)\right|=\left|T_{n}(z)\right|
$$

by Lemma 3. Since, in (11) equality holds if and only if $\rho_{k}=R_{k}, \psi_{k}=\varphi_{k}$ for $k=0, \ldots, n$, it is easily seen from the above proof that $|f(z)|<\left|T_{n}(z)\right|$ for all $z \in \mathcal{H}_{R}$ with $R \geq \xi_{n}$ unless $f(z) \equiv \pm T_{n}(z)$.

Now let $1 \leq k \leq n$. Assume that $\xi_{j} \neq x_{j}$ for some $j$. Then $\xi_{n} \neq x_{n}$ and $f(z)$ is not identically equal to $\pm T_{n}(z)$. Consequently, $|f(z)|<\left|\lambda T_{n}(z)\right|$ for all $z \in \mathcal{H}_{R}$ and all $\lambda \in \mathbb{C}$ such that $|\lambda| \geq 1$. This means that the polynomial $p(z):=$ $f(z)-\lambda T_{n}(z) \neq 0$ in $\mathcal{H}_{R}$. Hence, there exists a positive number $\delta$ such that all the zeros of $p$ lie in the half-plane $\operatorname{Re}(z) \leq \xi_{n}-\delta$. By the Gauss-Lucas theorem $[4, p .84]$ all the zeros of $p^{(k)}$, if any, also lie in the same half-plane. It follows that $f^{(k)}(z)-\lambda T_{n}^{(k)}(z) \neq 0$ in $\mathcal{H}_{R}$ for all $\lambda \in \mathbb{C}$ such that $|\lambda| \geq 1$. This is possible only if $\left|f^{(k)}(z)\right|<\left|T_{n}^{(k)}(z)\right|$ for all $z \in \mathcal{H}_{R}$. Indeed, if we had $\left|f^{(k)}\left(z_{0}\right)\right| \geq\left|T_{n}^{(k)}\left(z_{0}\right)\right|$ for some $z_{0} \in \mathcal{H}_{R}$, then with $\lambda_{0}=f^{(k)}\left(z_{0}\right) / T_{n}^{(k)}\left(z_{0}\right)$, which is of modulus $\geq 1$, we 
would have

$$
f^{(k)}\left(z_{0}\right)-\lambda_{0} T_{n}^{(k)}\left(z_{0}\right)=0
$$

contradicting the fact that $f^{(k)}(z)-\lambda T_{n}^{(k)}(z) \neq 0$ in $\mathcal{H}_{R}$ if $|\lambda| \geq 1$.

Remark 3. Let $x_{j}, \xi_{j}$ and $\mathcal{H}_{R}$ be as above. The argument used to prove Theorem $1^{*}$ shows that if $f$ is any polynomial of degree at most $n$, with real or complex coefficients, such that $\left|f\left(\xi_{j}\right)\right| \leq 1$ for $j=0,1, \ldots, n$ and $z_{0} \in \mathcal{H}_{R}$, then

$$
\left|f\left(z_{0}\right)\right| \leq\left|T_{n, z_{0}}\left(z_{0}\right)\right|
$$

where $T_{n, z_{0}}$ is the unique polynomial of degree $n$ satisfying the interpolation condition

$$
T_{n, z_{0}}\left(x_{j}\right)=(-1)^{n-j} \exp \left(i \arg \left(z_{0}-x_{j}\right)\right) \quad(j=0,1, \ldots, n) .
$$

Note that the extremal polynomial $T_{n, z_{0}}(z)$ may change with $z_{0}$. But clearly, $T_{n, z_{0}}(z) \equiv T_{n}(z)$, when $z_{0} \in \mathcal{H}_{R} \cap \mathbb{R}$.

\section{REFERENCES}

1. B. Bojanov, Elementary Proof of the Remez Inequality, Amer. Math. Monthly 100 (1993), pp. 483-485. CMP 93:11

2. P. Borwein \& T. Erdélyi, Polynomials and Polynomial Inequalities, Springer - Verlag, 1995. MR 97e:41001

3. G. Freud, Orthogonal Polynomials, Pergamon Press, Oxford, 1971.

4. E. Hille, Analytic Function Theory, vol. I, Blaisdel Publishing Company, New York, 1959. MR 21:6415

5. V.A. Markov (W.A. Markov), Über polynôme die in einem gegebenem Intervalle möglichst wenig von Null abweichen, Math. Ann. 77 (1916), pp. 213-258.

6. G. V. Milovanović, D. S. Mitrinović, Th. M. Rassias, Topics in Polynomials: Extremal Problems, Inequalities, World Scientific, Singapore, 1994. MR 95m:30009

7. I.P. Natanson, Constructive Function Theory, vol. I, Frederick Ungar Publishing Co., Inc., New York, 1964. MR 33:4529a

8. E.J. Remez, Sur une propriété des polynômes de Tchebycheff, Comm. Inst. Sci. Khar-kow 13 (1936), pp. 93-95.

9. T.J. Rivlin, Chebyshev Polynomials: From Approximation Theory to Algebra and Number Theory, 2nd ed., Wiley \& Sons, New York, 1990. MR 92a:41016

Department of Mathematics, University of Sofia, James Boucher 5, 1126 Sofia, BulGARIA

E-mail address: dryanovd@fmi.uni-sofia.bg

Département de Mathématiques et de Statistique, Université de Montréal, Montréal, CANADA H3C $3 \mathrm{~J} 7$

E-mail address: rahmanqi@ere.umontreal.ca 